\title{
Efeito da variação estequiométrica na formação de ferritas BaM produzidas pelo método SHS e pelo método cerâmico tradicional
}

\section{(Effect of stoichiometric variation on the BaM ferrite production by SHS and traditional ceramic processes)}

\author{
M. T. Yasuda , H. L. Hasegawa ${ }^{1,2}$, P. I. Paulin F. ${ }^{I}$, M. R. Morelli ${ }^{1}$ \\ ${ }^{1}$ Departamento de Engenharia de Materiais - DEMa, Universidade Federal de S. Carlos - UFSCar \\ Rod. Washington Luiz, km 235, S. Carlos, SP 13565-905 \\ ${ }^{2}$ ECIL Met Tec Ltda. \\ R. Benjamim S. Baldy 2001, Piedade, SP 18170-000 \\ marcioya@iris.ufscar.br
}

\section{Resumo}

As ferritas do tipo BaM são cerâmicas ferrimagnéticas, usualmente aplicadas em sistemas de gravação magnética e como magnetos duros. Possuem alta estabilidade química, resistência a corrosão e baixo custo de produção. Diante da sua crescente importância, foi estudado o efeito da contribuição do excesso de bário no processamento e formação da fase ferrita BaM por duas distintas rotas: processamento por mistura de óxidos e a síntese por reação de combustão (método SHS) com o uso de carbonato e nitrato de bário. Duas relações de concentração entre bário e ferro foram utilizadas, uma estequiométrica $\left(\mathrm{n}=\mathrm{Fe}_{2} \mathrm{O}_{3} / \mathrm{BaO}=6\right)$ e uma outra não estequiométrica $\left(\mathrm{n}=\mathrm{Fe}_{2} \mathrm{O}_{3} / \mathrm{BaO}=5,45\right)$. Os pós processados com excesso de bário resultaram na presença da fase intermediária de formação $\mathrm{BaFe}_{2} \mathrm{O}_{4}$, a qual permanece após altas temperaturas de calcinação e mesmo após a sinterização de compactos obtidos a partir dos pós calcinados. As conclusões foram baseadas em difratogramas de raios $\mathrm{X}$ das ferritas $\mathrm{BaM}$ em pó após calcinação e nas amostras conformadas e sinterizadas, além de imagens obtidas por microscopia eletrônica de varredura. Os resultados de medidas magnéticas indicam a diminuição da coercividade, da indução magnética remanente e dos valores de $\mathrm{BH}_{\mathrm{Max}}$ dos pós obtidos com a contribuição do excesso de bário, provavelmente devido à presença da fase intermediária não magnética. Este efeito negativo foi mais efetivo nos pós processados pelo método SHS, chegando a uma redução de $28,8 \%$ no valor de coercividade e $35,1 \%$ no valor de $\mathrm{BH}_{\text {Maxx }}$. Os pós de ferritas $\mathrm{BaM}$ obtidos pelo método cerâmico tradicional apresentaram maiores valores de coercividade $(4,28 \mathrm{kOe})$ e $\mathrm{BH}_{\text {Máx }}(0,87$ $\mathrm{MOe}$ emu/g) e a influência do excesso de bário na formulação foi menos prejudicial sobre suas propriedades magnéticas.

Palavras-chave: hexaferrita, síntese de pós, reação de combustão.

Abstract

BaM ferrite is a ferrimagnetic ceramic usually applied in high density recording media and as permanent magnet. This magnetic material shows high chemical stability, corrosion resistance and low production cost. This work shows the effects of barium excess on the BaM ferrite processing and on their magnetic properties. The use of barium excess had been used in some of ferrite industries to avoid the hematite and magnetite phase on the final products. In this work, barium ferrites were produced by traditional ceramic route and by combustion reaction (SHS) using barium nitrate and also barium carbonate. Two relations between barium and iron were used in all BaM ferrite processing, one stoichiometric $\left(n=\mathrm{Fe}_{2} \mathrm{O}_{3} / \mathrm{BaO}=6\right)$ and another non-stoichiometric $\left(n=\mathrm{Fe}_{2} \mathrm{O}_{3} / \mathrm{BaO}=5.45\right)$. All powders were submitted to different calcination conditions and analyzed by scanning electron microscopy, X-ray diffraction and magnetic characteristics. The presence of barium excess on the BaM ferrite resulted in the presence of the nonmagnetic BaFe $\mathrm{O}_{4}$ phase that remains after the calcination and sintering processes. The $\mathrm{BaFe}_{2} \mathrm{O}_{4}$ phase is deleterious to the powder magnetic properties such as coercivity, remanence magnetic induction and $B H_{M a x}$. This damage was more significant on the BaM ferrite processed by SHS process, where the coercivity decreased in $28.8 \%$ and the $\mathrm{BH}_{\mathrm{u}}$ in $35,1 \%$. The barium ferrite powder produced by traditional ceramic route shows high values of coercivity $(4.28 \mathrm{kOe})$ and $\mathrm{BH}_{\mathrm{Max}}\left(0.87 \mathrm{MOe} \mathrm{e}_{\mathrm{emu}} \mathrm{g}\right)$, and the effect on the magnetic properties of barium excess was not so harmful than it was in the ferrite powders produced by SHS.

Keywords: hexaferrites, traditional ceramic processes, combustion reaction.

\section{INTRODUÇ̃̃O}

As ferritas do tipo BaM, também conhecidas como hexaferritas de bário $\left(\mathrm{BaFe}_{12} \mathrm{O}_{19}\right)$, têm sido alvo de estudos desde a década de 50 , quanto às suas propriedades magnéticas, e continuam sendo abordadas [1-4]. Inicialmente o uso das ferritas BaM se restringiam a magnetos permanentes e em meios de gravação magnética. Hoje, além dessas aplicações, as hexaferritas estão sendo estudadas quanto a suas novas aplicações 
promissoras como, por exemplo, na absorção de radiação eletromagnética e gravações de alta densidade [5-6]. A alta coercividade, resistência a corrosão, estabilidade química e baixo custo de produção, têm levado as ferritas $\mathrm{BaM}$ a ocupar uma parte significativa do mercado de materiais magnéticos mesmo após o surgimentos das ligas ferromagnéticas de terras-raras como o SmCo e o $\mathrm{NdFeB}$

O processamento via o método cerâmico tradicional, também conhecido como mistura de óxidos, é o principal processo de produção utilizado na fabricação de ferritas $\mathrm{BaM}$ pelas indústrias, devido ao baixo custo em grande escala. Diante deste fato, alguns trabalhos científicos têm abordado os efeitos das etapas do processamento via mistura de óxidos nas propriedades magnéticas, assim como técnicas mais sofisticadas de processamento cerâmico como, por exemplo, o método SHS (Self-propagation High-temperature Synthesis), também conhecido como reação de combustão, o método de cristalização vítrea, o método sol-gel, a técnica de co-precipitação química, entre outros [7-12].

No processamento das ferritas BaM pelo método cerâmico tradicional utilizam-se óxidos e carbonatos onde duas ou três etapas ocorrem para a formação da fase hexaferrita, dependendo do uso do carbonato de bário ou óxido de bário, como apresentado nas equações A a C. A segunda etapa é descrita pela formação da fase intermediária monoferrita $\mathrm{BaFe}_{2} \mathrm{O}_{4}$, o qual tende a reagir com as hematitas restantes para finalmente formar a fase ferrita BaM. A relação estequiométrica desta reação é de $\mathrm{n}=6$, onde $\mathrm{n}=$ $\mathrm{Fe}_{2} \mathrm{O}_{3} / \mathrm{BaO}$ é o fator de estequiometria.

$$
\begin{aligned}
& \mathrm{BaCO}_{3} \rightarrow \mathrm{BaO}+\mathrm{CO}_{2} \\
& \mathrm{BaO}+\mathrm{Fe}_{2} \mathrm{O}_{3} \rightarrow \mathrm{BaFe}_{2} \mathrm{O}_{4} \\
& \mathrm{BaFe}_{2} \mathrm{O}_{4}+5 \mathrm{Fe}_{2} \mathrm{O}_{3} \rightarrow \mathrm{BaFe}_{12} \mathrm{O}_{19}
\end{aligned}
$$

$\mathrm{O}$ interesse em obter a melhora das propriedades magnéticas das ferritas BaM, como por exemplo a coercividade e a magnetização de saturação, levantou a questão sobre o uso de um excesso de bário $(\mathrm{n}<6)$ no processo de produção das ferritas $\mathrm{BaM}$ e as conseqüências deste uso [9,12-14]. Surig et al [13] tem mostrado que as melhores propriedades magnéticas das ferritas $\mathrm{BaM}$ têm sido obtidas com valores de $n$ entre 5 e 5,5, para diferentes técnicas de processamento. Estes valores se encontram dentro da região de uma fase sólida de composição $\mathrm{BaFe}_{12} \mathrm{O}_{19}$ no diagrama de fases, como apresentado na Fig. 1. No diagrama, a região de fase sólida da ferrita BaM está compreendida entre 83,29\% em mol de $\mathrm{Fe}_{2} \mathrm{O}_{3}(\mathrm{n}=4,98)$ e $85,71 \%(\mathrm{n}=6)$.

Foi mostrado que a razão da melhora das propriedades magnéticas produzidas com valores de n entre 5 e 5,5 é a ocorrência de maior grau de ocupação dos sítios octaédricos

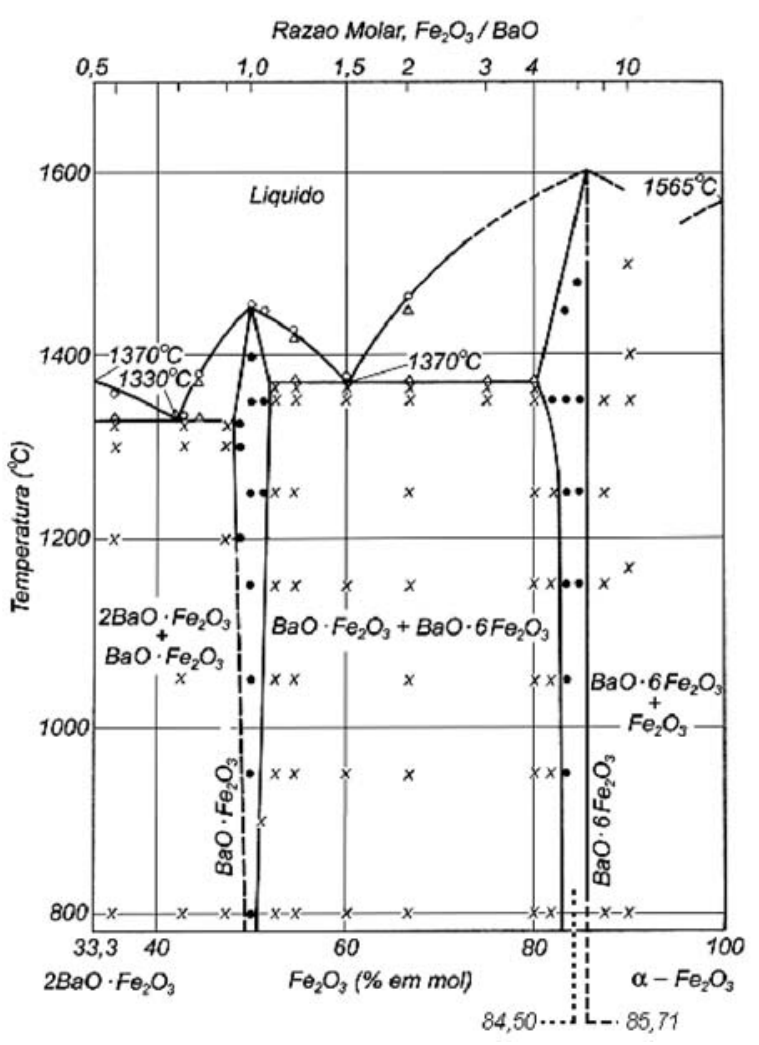

Figura 1: Diagrama de fases $\mathrm{BaO}-\alpha-\mathrm{Fe}_{2} \mathrm{O}_{3}$ [15], que apresenta as composições estequiométrica (---) $(85,71 \%, n=6)$ e nãoestequiométrica (---) $(84,50 \%, n=5,45)$ utilizadas neste trabalho. Neste diagrama $\boldsymbol{x}$ representa uma região de duas fases sólidas, • uma região de uma fase sólida, $\Delta$ uma região de fases sólida e líquida coexistindo e o uma região de uma fase líquida.

[Figure 1: Phase diagram of $\mathrm{BaO}-\alpha-\mathrm{Fe}_{2} \mathrm{O}_{3}$ [15]. The positions identified by (---) represent a non-stoichiometric composition (84,50\%, $n=5.45)$ and (---) a stoichiometric composition $(85,71 \%, n=6)$ used in this work. In this diagram $\boldsymbol{x}$ represents $a$ region with two solid phases, $\cdot$ a region with one solid phase, $\Delta a$ region composed by a solid and a liquid phase coexisting together, and $\mathrm{O}$ a region composed by one liquid phase.]

pelos íons ferro $(50 \%$ para $\mathrm{n}=5,25)$ na rede cristalina da ferrita BaM $[13,14]$, após a "lavagem" do material resultante, obtido pelo método sol-gel, com ácido clorídrico a fim de se eliminar a fase intermediária monoferrita $\mathrm{BaFe}_{2} \mathrm{O}_{4}$.

O objetivo deste trabalho é a avaliação dos efeitos do uso do excesso de bário em $0,1 \%$ em mol, ou seja, $\mathrm{n}=5,45$ (composição central de $84,50 \%$ na região de uma fase sólida no diagrama da Fig. 1) nas propriedades magnéticas das ferritas BaM processadas pelo método cerâmico tradicional e pelo método SHS sem a etapa de "lavagem" com ácido clorídrico.

\section{PROCEDIMENTO EXPERIMENTAL}

As hexaferritas de bário foram processadas por duas distintas rotas: pelo método cerâmico tradicional e pelo método SHS. No processamento via método cerâmico 
tradicional, uma mistura a úmido dos reagentes $\left(\mathrm{BaCO}_{3}\right.$ $99,9 \%$, Mallinckodt, e $\alpha-\mathrm{Fe}_{2} \mathrm{O}_{3} 99,2 \%$, J.T. Baker), foi feita em um moinho de bolas por $4 \mathrm{~h}$, em um recipiente de polietileno contendo álcool etílico e bolas de zircônia na proporção em massa 4:1:1 (bolas:mistura:álcool). A amostra preparada na relação estequiométrica $(n=6)$ é identificada neste trabalho por Mix 1 e a preparada fora da estequiometria $(n=5,45)$ por Mix 1,1 .

As reações de combustão no método SHS foram feitas com uréia $\left(\mathrm{CO}\left(\mathrm{NH}_{2}\right)_{2} 99,5 \%\right.$, Synth), como elemento combustível para a reação, em um recipiente de sílica vítrea sobre uma placa aquecedora. $\mathrm{Na}$ reação de combustão utilizando apenas nitratos $\left(\mathrm{Ba}\left(\mathrm{NO}_{3}\right)_{2} 99,9 \%\right.$, Modern Chemistry e $\mathrm{Fe}\left(\mathrm{NO}_{3}\right)_{3} \cdot 9 \mathrm{H}_{2} \mathrm{O} 98,9 \%$, Ecibra) foi usada a seguinte relação entre os componentes $\left(\mathrm{Ba}\left(\mathrm{NO}_{3}\right)_{2}: \mathrm{Fe}\left(\mathrm{NO}_{3}\right)_{3} \cdot 9 \mathrm{H}_{2} \mathrm{O}\right.$ : $\left.\mathrm{CO}\left(\mathrm{NH}_{2}\right)_{2}\right):: 1: 12: 31,7$ e $1,1: 12: 31,9$, denominados $\mathrm{Ni} 1(\mathrm{n}=$ 6) e Ni $1,1(n=5,45)$, respectivamente.

$\mathrm{O}$ processamento da ferrita BaM pelo método SHS com o uso do carbonato de bário foi feita com ácido cítrico $\left(\mathrm{C}_{6} \mathrm{H}_{8} \mathrm{O}_{7} 99,5 \%\right.$, Synth $)$ para a de-carbonificação, além da presença de nitrato de ferro e uréia. As relações dos componentes para esta reação foram $\left(\mathrm{BaCO}_{3}\right.$ : $\left.\mathrm{Fe}\left(\mathrm{NO}_{3}\right)_{3} \cdot 9 \mathrm{H}_{2} \mathrm{O}: \mathrm{CO}\left(\mathrm{NH}_{2}\right)_{2}\right):: 1: 12: 30$ e $1,1: 12: 30$, denominados a seguir por $\mathrm{C} 1(\mathrm{n}=6)$ e $\mathrm{C} 1,1(\mathrm{n}=5,45)$, respectivamente.

As calcinações dos pós em ar atmosférico foram feitas em um forno elétrico em diferentes condições de temperatura e tempo de patamar de queima. Os pós resultantes das distintas rotas de processamento foram investigados quanto às fases presentes com um difratômetro de raios $\mathrm{X}$ equipado com monocromador de grafite (Rigaku Geigerflex). Quanto à morfologia e tamanho médio de partículas, foi usado um microscópio eletrônico de varredura (Philips XL 30 FEG). Para a identificação das fases resultantes da sinterização das amostras $\left(1200^{\circ} \mathrm{C}\right.$ por $\left.2 \mathrm{~h}\right)$ foi feita a prensagem uniaxial de $20 \mathrm{MPa}$ dos pós em pastilhas.

As medidas magnéticas em temperatura ambiente das amostras em pó foram feitas em um permeâmetro magnético (Magnetech P01) para a obtenção da curva de desmagnetização. A curva de histerese magnética não foi obtida devido o limite do campo magnético do permeâmetro magnético não ser suficiente para atingir a magnetização de saturação dos pós (8,57 kOe). Para a curva de desmagnetização das amostras, a magnetização de saturação dos pós foi alcançada com o uso de um equipamento de indução magnética (Magnet Charger 1000 Joules) que aplica, através de pulsos, um campo nominal de 3,7 Tesla.

\section{RESULTADOS E DISCUSSÃO}

Os pós calcinados processados pelas distintas rotas de processamento com o excesso de bário $(n=5,45)$ resultaram na presença da fase monoferrita $\mathrm{BaFe}_{2} \mathrm{O}_{4}$, a qual sua concentração foi diminuída de acordo com o aumento da temperatura de calcinação. As Figs. 2 a 4 apresentam os difratogramas de raios $\mathrm{X}$ após calcinação em $1100{ }^{\circ} \mathrm{C}$ por $2 \mathrm{~h}$ e destas mesmas composições após sinterização a $1200^{\circ} \mathrm{C}$ por 2 horas.

A presença da fase intermediária é notada mesmo após o favorecimento da difusão durante a sinterização com o aumento do contato entre as partículas e a alta temperatura de sinterização.. A relação de n utilizada está dentro da região de uma fase sólida do diagrama de fases da Fig. 1, a qual se esperava estar presente apenas a fase ferrita BaM devido a temperatura utilizada e segundo Goto e Takada [15], autores originais do diagrama. Segundo esses autores, a fase intermediária se dissolve para uma extensão da fase ferrita $\mathrm{BaM}\left(\mathrm{BaO} \cdot 6 \mathrm{Fe}_{2} \mathrm{O}_{3}\right)$ e a solubilidade da fase $\mathrm{Fe}_{2} \mathrm{O}_{3}$ não foi encontrada por eles [15]. A dissociação mencionada pode explicar a migração dos átomos da fase monoferrita para a contínua formação da fase ferrita BaM com o aumento da temperatura aplicada para a calcinação e/ou sinterização.

As micrografias da Fig. 5 ilustram a forma e características das partículas constituintes dos pós preparados com a relação estequiométrica de $n=5,45$ e $\mathrm{n}=6$ e pelos os diferentes métodos de processamento. As imagens foram obtidas no mesmo aumento de 10.000 vezes.

Pode se observar nas imagens da Fig. 5 uma distribuição mais homogênea e de menor tamanho médio de partículas (aproximadamente $400 \mathrm{~nm}$ ) nos pós obtidos pela mistura de óxidos, em relação aos pós obtidos pelo método SHS. Os pós preparados via o método SHS apresentam além de

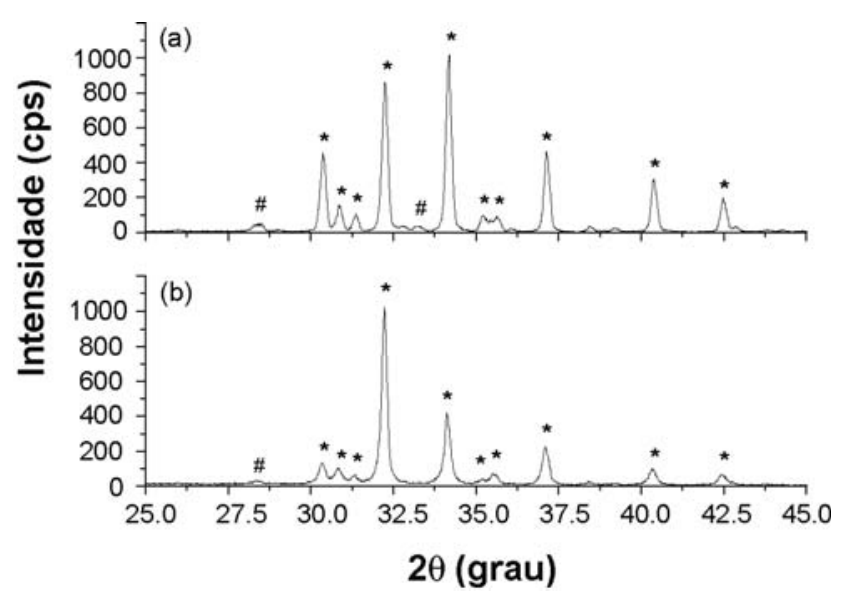

Figura 2: (a) DRX do pó de ferrita $B a M$ obtida pelo método cerâmico tradicional e de fração estequiométrica de $n=5,45$, Mix 1,1 calcinada a $1000{ }^{\circ} \mathrm{C}$ por $6 \mathrm{~h}$ e (b) da pastilha de Mix 1,1 sinterizada a $1200{ }^{\circ} \mathrm{C}$ por $2 \mathrm{~h}$. O símbolo * representa as reflexões basais da hexaferrita de bário $\left(\mathrm{BaFe}_{12} \mathrm{O}_{19}\right)$ e\# representa as reflexões basais da fase intermediária $\mathrm{BaFe}_{2} \mathrm{O}_{4}$.

[Figure 2: (a) XRD of BaM ferrite powder obtained by traditional ceramic route and prepared with non-stoichiometric composition (n = 5.45), Mix 1,1 calcined at $1000^{\circ} \mathrm{C}$ for $6 \mathrm{hs}$ and (b) XRD of pellet of Mix 1,1 sintered at $1200^{\circ} \mathrm{C}$ for $2 \mathrm{hs}$. The symbol * represents basal reflections of barium hexaferrite $\left(\mathrm{BaFe}_{12} \mathrm{O}_{19}\right)$ and \# represents basal reflections of intermediate phase $\mathrm{BaFe}_{2} \mathrm{O}_{4}$.] 


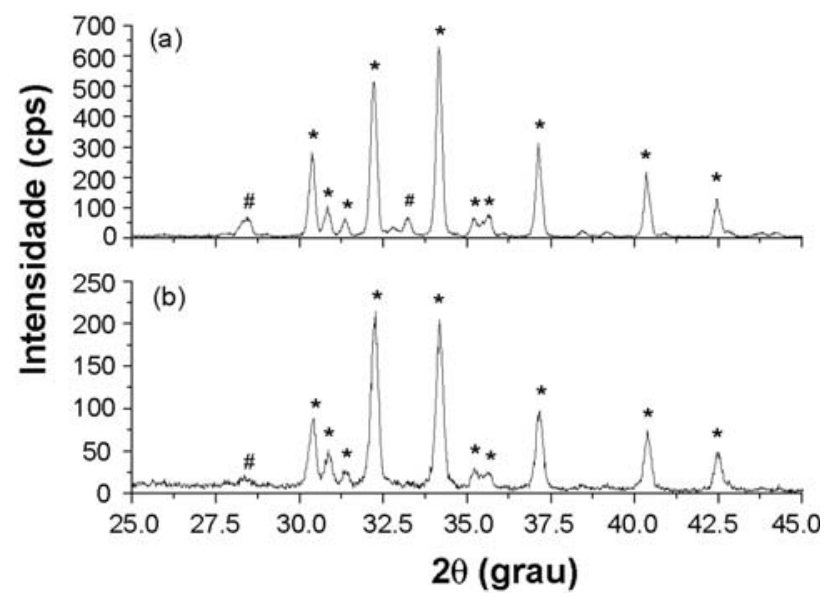

Figura 3: (a) DRX do pó de hexaferrita de bário obtida pelo método SHS com o uso somente de nitratos e de fração estequiométrica de $n=5,45$, Ni 1,1 calcinada a $1100{ }^{\circ} \mathrm{C}$ por $2 \mathrm{~h}$ e (b) da pastilha de Ni 1,1 sinterizada a $1200{ }^{\circ} \mathrm{C}$ por $2 \mathrm{~h}$. $\mathrm{O}$ símbolo * representa as reflexões basais da hexaferrita de bário $\left(\mathrm{BaFe}_{12} \mathrm{O}_{19}\right)$ e \# representa as reflexões basais da fase intermediária $\mathrm{BaFe}_{2} \mathrm{O}_{4}$.

[Figure 3: (a) XRD of barium hexaferrite powder obtained by SHS process using only nitrates and prepared with nonstoichiometric composition $(n=5.45)$, Ni 1,1 calcined at $1100^{\circ} \mathrm{C}$ for $2 \mathrm{~h}$ and (b) XRD of pellet of Ni 1,1 sintered at $1200^{\circ} \mathrm{C}$ for $2 \mathrm{~h}$. The symbol * represents basal reflections of barium hexaferrite $\left(\mathrm{BaFe}_{12} \mathrm{O}_{10}\right)$ and \# represents basal reflections of intermediate phase $\mathrm{BaFe}_{2} \mathrm{O}_{4}$.]

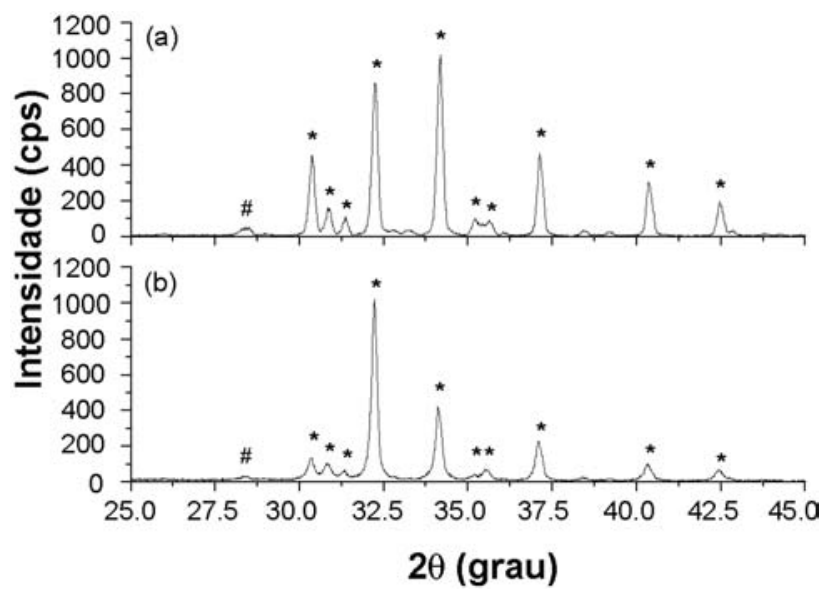

Figura 4: (a) DRX do pó de hexaferrita de bário obtida pelo método SHS com o uso de carbonato de bário e de fração estequiométrica de $n=5,45$, C 1,1 calcinada a $1100{ }^{\circ} \mathrm{C}$ por $2 \mathrm{~h}$ e (b) da pastilha de C 1,1 sinterizada a $1200{ }^{\circ} \mathrm{C}$ por $2 \mathrm{~h}$. O símbolo * representa as reflexões basais da hexaferrita de bário $\left(\mathrm{BaFe}_{12} \mathrm{O}_{19}\right)$ e \# representa as reflexões basais da fase intermediária $\mathrm{BaFe}_{2} \mathrm{O}_{4}$.

[Figure 4: (a) XRD of barium hexaferrite powder obtained by SHS process using Barium carbonate and prepared with nonstoichiometric composition $(n=5.45), C 1,1$ calcined at $1100^{\circ} \mathrm{C}$ for $2 \mathrm{~h}$ and (b) XRD of pellet of C 1,1 sintered at $1200^{\circ} \mathrm{C}$ for $2 \mathrm{~h}$. The symbol * represents basal reflections of barium hexaferrite $\left(\mathrm{BaFe}_{12} \mathrm{O}_{19}\right)$ and \# represents basal reflections of intermediate phase $\mathrm{BaFe}_{2} \mathrm{O}_{4}$ ] tamanho médio de partículas maiores (aproximadamente $800 \mathrm{~nm}$ ) um maior grau de aglomeração, diferentemente dos pós Mix 1 e Mix 1,1. Tanto o tamanho médio das partículas como a aglomeração ocasionam um alteração nas propriedades magnéticas do pó como pode se observar nos resultados apresentados a seguir.

Com o intuito de comparação, foi realizado em conjunto com as amostras processadas pelo método SHS e mistura de óxidos, medidas magnéticas em uma amostra comercial. A Fig. 6a ilustra as curvas de histerese magnética e de desmagnetização da amostra comercial, obtidas pelo permeâmetro magnético. A desmagnetização foi realizada após a amostra ser submetida a um pulso de magnetização de 3,7 Tesla nominal. O permeâmetro magnético pode ser utilizado para obter curvas de histerese magnética a temperatura ambiente, porém o limite de campo magnético (H) aplicável por este equipamento é limitado o que, para determinadas amostras, é insuficiente para se alcançar a magnetização de saturação $\left(\sigma_{\mathrm{S}}\right)$ da amostra magnética. Um exemplo desta condição é apresentado na figura 6 (a). Das medidas de desmagnetização é possível se obter além da indução magnética remanente $\left(\mathrm{B}_{\mathrm{R}}\right)$, a coercividade $\left(\mathrm{H}_{\mathrm{C}}\right)$ e o valor do produto de $\mathrm{B}$ por $\mathrm{H}$ máximo $\left(\mathrm{BH}_{\text {Máx }}\right)$. As curvas de desmagnetização de todas as amostras são estudadas e apresentadas na Fig. 6 b.

A Fig. 7 mostra o comportamento do produto entre a os valores de indução magnética da amostra e o campo magnético aplicado sobre esta $(\mathrm{BxH})$. Os pontos apresentados na Fig. 7 foram gerados pelo ajuste das curvas experimentais da Fig. 6b por uma expressão exponencial associada. Os valores de indução magnética remanente, coercividade e $\mathrm{BH}_{\text {Máx }}$ foram extraídas dos gráficos das Figs. 6b e 7 e são expostos na Tabela I.

As propriedades magnéticas dos pós de ferrita BaM indicam uma influência negativa da fase monoferrita não magnética como esperado. Isto foi mais evidente para as amostras preparadas pelo método de reação por combustão, sendo mais discrepante para as reações com o uso de nitratos, chegando a uma redução de $28,8 \%$ no valor de coercividade e $35,13 \%$ no valor de $\mathrm{BH}_{\text {Máx }}$. Para os pós Mix 1 e Mix 1,1 as curvas de desmagnetização praticamente coincidiram e suas propriedades magnéticas foram bastante próximas, indicando que a quantidade remanescente da fase intermediária na amostra Mix 1,1 afetou pouco suas propriedades, chegando até a apresentar um valor de indução magnética remanente levemente maior que a amostra Mix 1 de relação estequiométrica de $\mathrm{n}=6$. Os valores mais elevados de coercividade, indução remanente e $\mathrm{BH}_{\text {Máx }}$ das amostras processadas pelo método cerâmico tradicional em relação aos pós preparados pelo método SHS se deve também ao menor tamanho médio das partículas assim como o menor grau de aglomeração destas. Isto é válido para partículas com tamanho médio abaixo de $1 \mu \mathrm{m}$, onde as partículas apresentam monodomínios magnéticos, e acima da escala nanométrica, onde elas começam a exibir um comportamento superparamagnético $[4,16]$. 


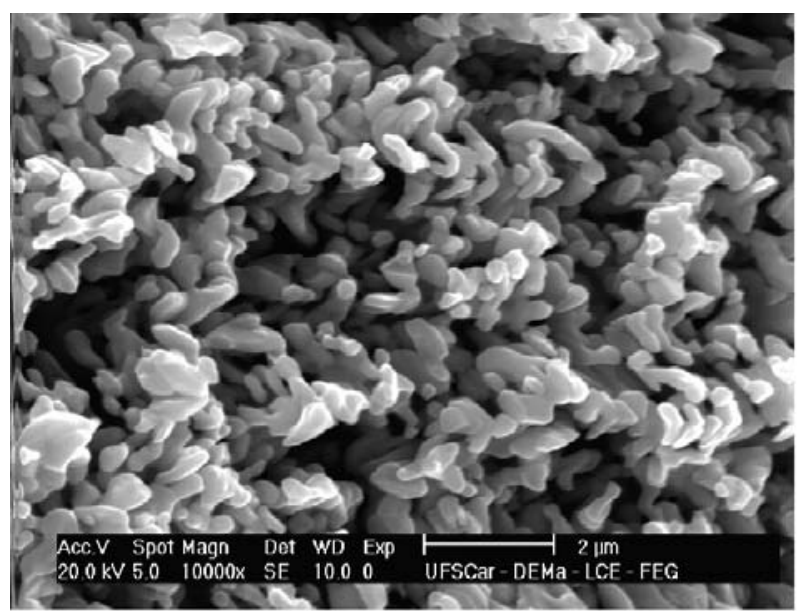

(a) Mix 1

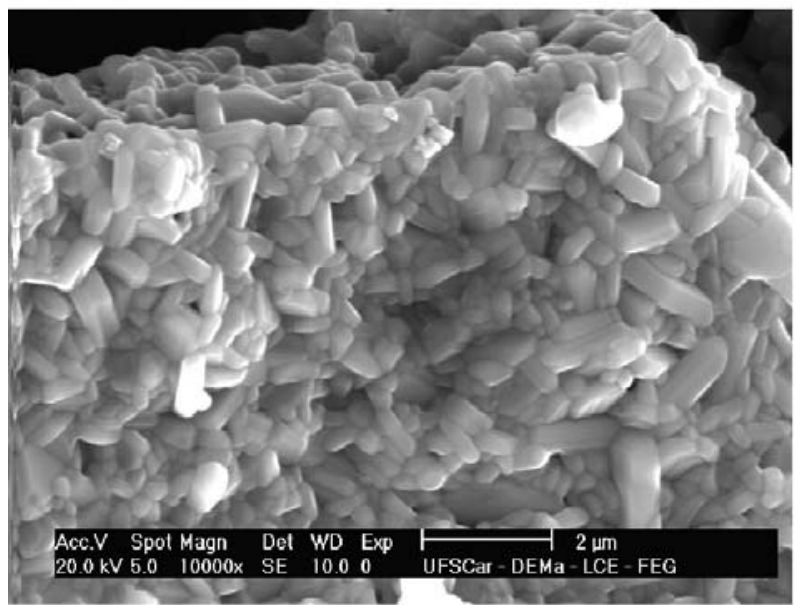

(c) $\mathrm{Ni} 1$

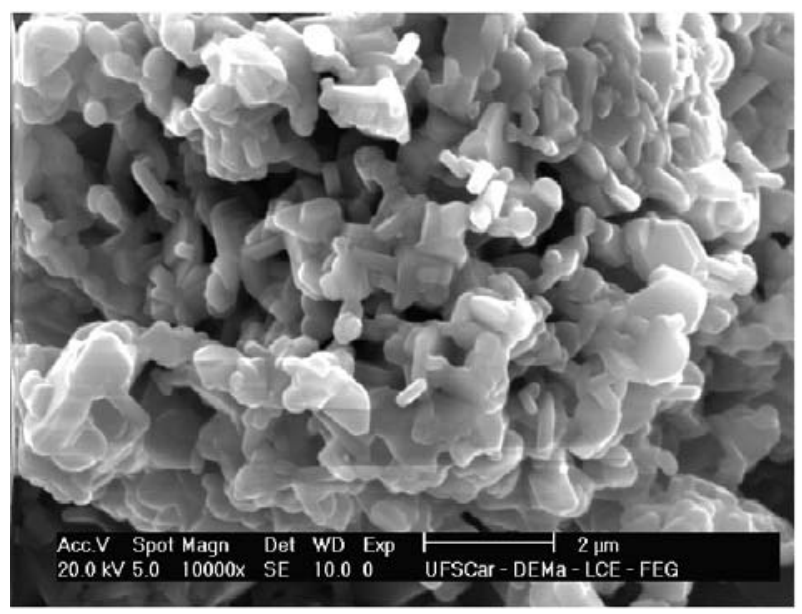

(e) C 1

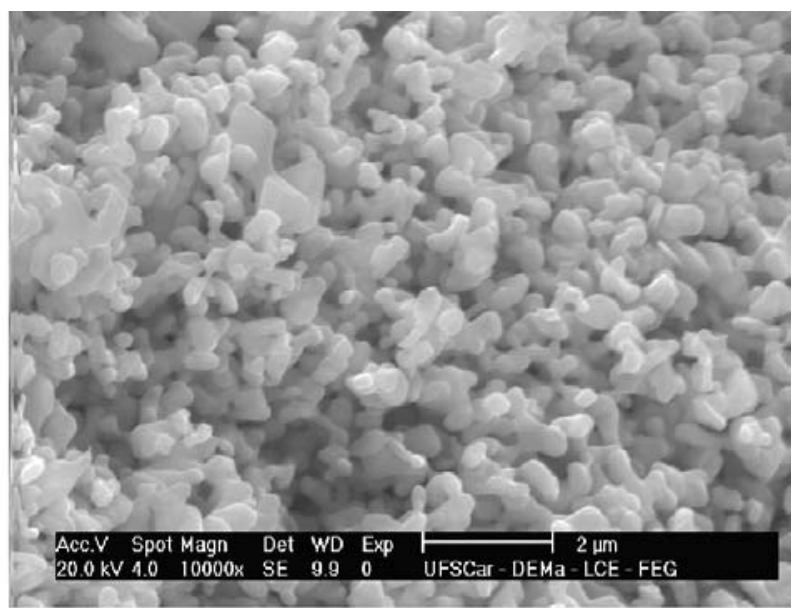

(b) $\operatorname{Mix} 1,1$

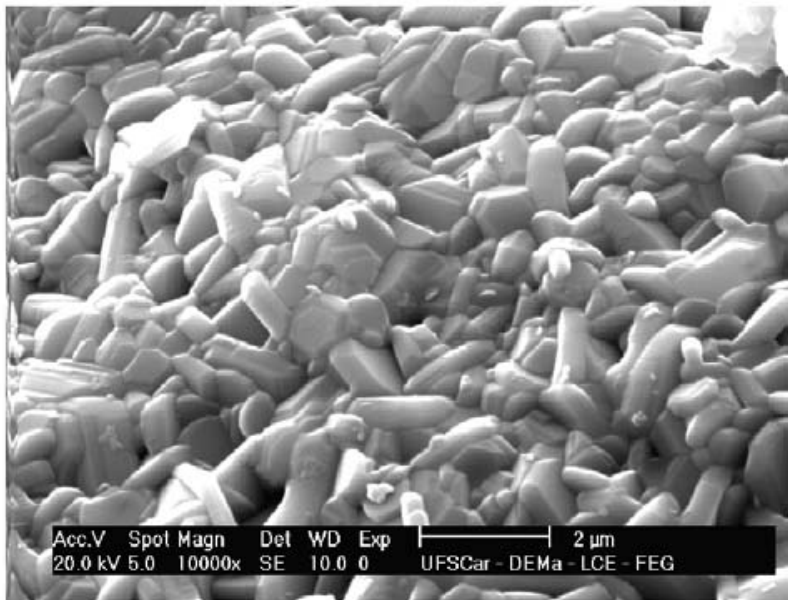

(d) $\mathrm{Ni} 1,1$

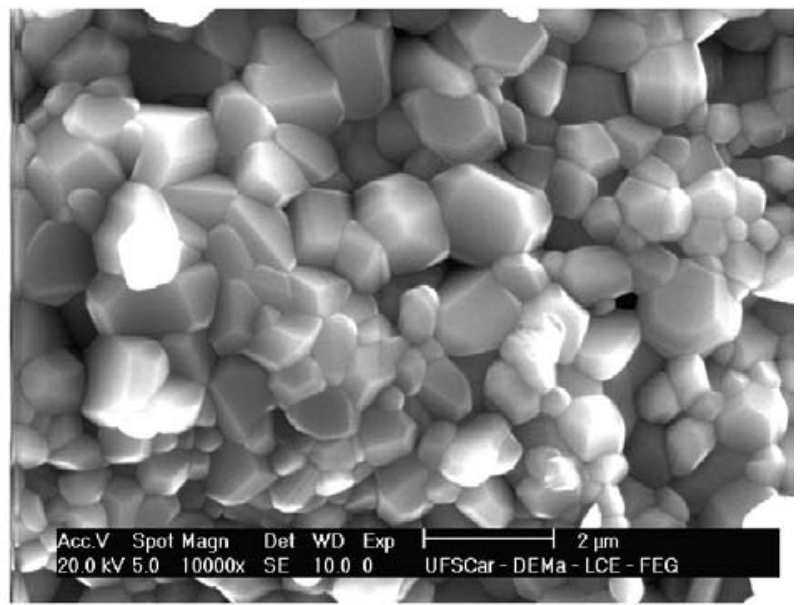

(f) C 1,1

Figura 5: Micrografias obtidas por microscopia eletrônica de varredura de diferentes composições de ferrita BaM em pó (estequiométrica e não estequiométrica) preparadas por mistura de óxidos, (a) e (b), e reação de combustão (c), (d), (e) e (f)).

[Figure 5: SEM micrographs of different BaM ferrite powders (stoichiometric and non-stoichiometric) prepared by traditional ceramic process, (a) and (b), and SHS process, (c), (d), (e) and (f)).] 

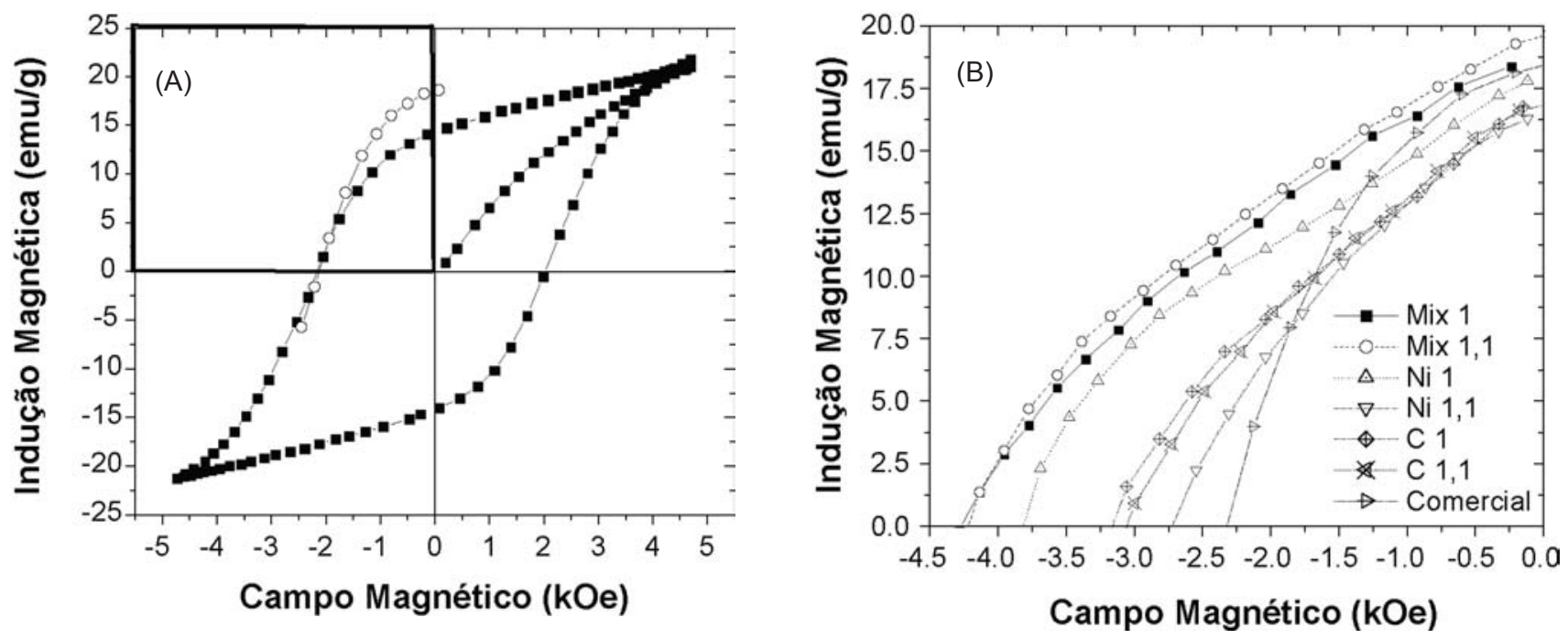

Figura 6: (a) Curva de histerese magnética obtida da amostra comercial (-m-) e curva de desmagnetização (-o-) para a mesma após magnetização de 3,7 Tesla. (b) Curvas de desmagnetização para as diversas ferritas $B a M$, inclusive a comercial apresentada no item (a). Obs. Todas as medidas magnéticas foram obtidas a temperatura ambiente.

[Figure 6: (a) Magnetic hysteresis of commercial sample (-n-) and demagnetization measurement (-o-) of the same sample after maximum magnetization of 3.7 Tesla. (b) Demagnetization measurements of all BaM ferrite powder samples. Obs. All magnetic measurements were obtained at room temperature.]

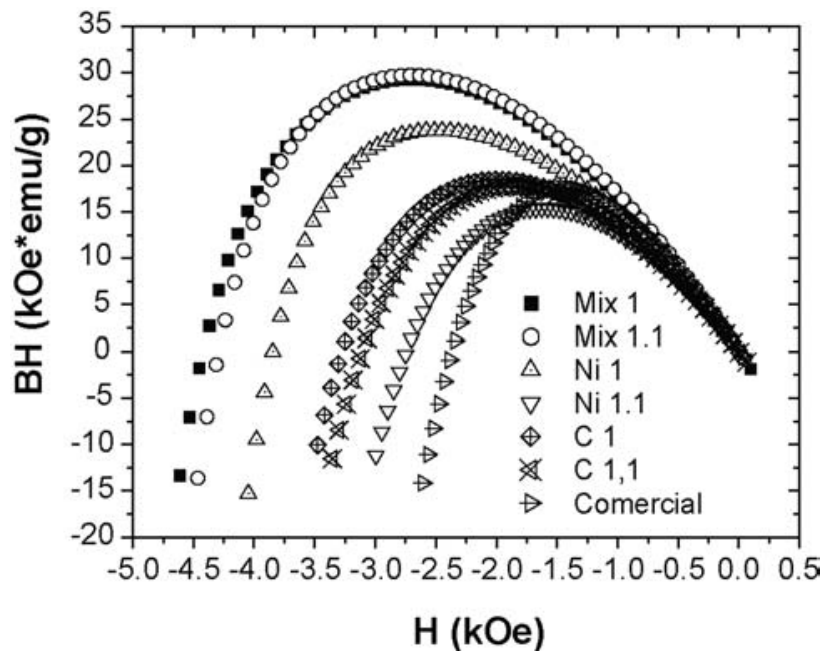

Figura 7: Curvas do produto entre a indução magnética sobre a amostra (B) e o campo magnetizante $(\mathrm{H})$ para as distintas amostras. Os pontos foram obtidos das curvas de desmagnetização apresentadas na Fig. 6a.

[Figure 7: Behavior of the multiplication between the sample magnetic induction (B) by magnetic field (H) for all samples. The points were obtained from demagnetization behaviors of figure 6b.]

\section{CONCLUSÕES}

O uso da relação estequiométrica $n=5,45$ resultou na coexistência da fase intermediária $\mathrm{BaFe}_{2} \mathrm{O}_{4}$ após a calcinação dos pós produzidos pelo método cerâmico tradicional e pelo método SHS. Esta fase permanece
Tabela I - Propriedades magnéticas das ferritas $B a M$ em pó obtidas dos gráficos das Figs.6a e 7.

[Table I - Magnetic properties of BaM ferrite powders obtained by results presented in Figs. 6a e 7.]

\begin{tabular}{cccc}
\hline Amostra & $\begin{array}{c}\mathrm{H}_{\mathrm{C}} \\
(\mathrm{kOe})\end{array}$ & $\begin{array}{c}\mathrm{B}_{\mathrm{R}} \\
(\mathrm{emu} / \mathrm{g})\end{array}$ & $\begin{array}{c}\mathrm{BH}_{\text {Máx }} \\
\left(\mathrm{MOe}^{\mathrm{e}} \mathrm{emu} / \mathrm{g}\right)\end{array}$ \\
\hline Mix 1 & 4,28 & 19,01 & 0,87 \\
Mix 1,1 & 4,21 & 19,62 & 0,88 \\
Ni 1 & 3,82 & 18,02 & 0,70 \\
Ni 1,1 & 2,72 & 16,52 & 0,46 \\
C 1 & 3,17 & 17,23 & 0,56 \\
C 1,1 & 3,05 & 16,88 & 0,53 \\
Comercial & 2,32 & 18,30 & 0,52 \\
\hline
\end{tabular}

presente mesmo após a sinterização de pastilhas prensadas com estes mesmos pós já calcinados, apesar do diagrama de fases descrito por Goto e Takada [15] apresentar uma região de uma fase sólida da ferrita BaM. Porém nota-se que esta fase tende a se dissolver para a fase ferrita BaM de acordo com a temperatura de calcinação e/ou a temperatura de tratamento térmico. $\mathrm{O}$ uso das relações estequiométricas $\mathrm{n}<6$ são favoráveis às propriedades magnéticas contanto que se elimine a fase intermediária não magnética, onde a fase $\mathrm{BaFe}_{2} \mathrm{O}_{4}$ foi prejudicial mesmo em pequenas concentrações. A relação estequiométrica $n=5,45$ resultou, em relação a relação estequiométrica $n=6$, na diminuição significativa da coercividade dos pós produzidos por ambos os processos de produção: método cerâmico tradicional (de 4,28 kOe para 4,21 kOe) e pelo método SHS (de 3,82 kOe 
para 2,72 kOe e de 3,17 kOe para 3,05 kOe). Os efeitos da variação estequiométrica nos processos de produção pelo método SHS demonstram ser mais prejudiciais sobre as propriedades magnéticas dos pós em relação ao método cerâmico tradicional, provavelmente devido a um maior tamanho de partículas (aproximadamente o dobro) e maior aglomeração, o que também resulta em menores valores de indução magnética remanente, coercividade e $\mathrm{BH}_{\text {Máx }}$.

\section{AGRADECIMENTOS}

Ao Programa de Pós-Graduação em Ciência e Engenharia de Materiais (PPG-CEM), ao Conselho Nacional de Desenvolvimento Científico e Tecnológico e a Fermag Ferritas Magnéticas Ltda.

\section{REFERÊNCIAS}

[1] J. J. Went, G. W. Rathenau, E. W. Gorter, G. W. Van Oosterhout, Phys. Rev. 86 (1952) 424.

[2] K. Haneda, A.H. Morrish, IEEE Trans. Magn. 25 (1989) 2597

[3] P. E. Garcia-Casillas, A. M. Beesley, D. Bueno, J. A. Matutes-Aquino, C. A. Martinez, J. Alloys Comp. 369 (2004) 185.

[4] M. Radwan, M. M. Rashad, M. M. Ressien, J. Mat. Process. Tech. 181 (2007) 106.

[5] R. C. Lima, J. C. S. Leandro, T. Ogasawara. Cerâmica.
49, 309 (2003) 44

[6] T. Nagata, T. Harasawa, M. Oyanagi, N. Abe, S. Saito, IEEE Trans. Magn. 42 (2006) 2312.

[7] J. Dufour, E. López-Vidriero, C. Negro, R. Latorre, E. M. Alcaá, F. López-Mateos, A. Formoso, Chem. Eng. Comm. 167 (1998) 227.

[8] I.P. Parkin, G.Elwin, M.V. Kuznetsov, Q.A. Pankhurst, Q. T. Bui, G. D. Forster, L. F. Barquín, A. V. Komarov, Y. G. Morozov. J. Mat. Process. Tech. 110 (2001) 239.

[9] P. Gornert, E. Sinn, W. Schuppel, IEEE Trans.Magn 26 (1990) 12.

[10] G. Mendonza-Suaréz, M. C. Cisneros-Morales, M. M. Cisneors-Guerrero, K. K. Johal, H. Mancha-Molinar, O. E. Ayala-Valenzuela, J. I. Escalante-Garcia, Mater. Chem. Phys. 77

[11] J. Matutes-Aquino, S. Diaz-Castañón, M. MirabalGarcía, S.A. Palomares-Sánches, Scripta Mater. 42 (2000) 295.

[12] X. Liu, J. Wang, L.-M, Gan, S.-C. Ng, J. Magn. Magn. Mat. 195 (1999) 452.

[13] C. Surig, K. A. Hempel, Ch. Sauer, J. Magn. Magn. Mat. 157/158 (1996) 268.

[14] C. Surig, D. Bonnemberg, K. A. Hempel, P. Karduck, H. J. Klaar, Ch. Sauer, Journal de Physique IV C1 (1997) 315.

[15] Y. Goto, T. Takada, J. Am. Cer. Soc. 43 (1960) 150. [16] V. Berbenni, A. Marini, N. J. Welham, P. Galinetto, M. C. Mozzat, J. Eur. Cer. Soc. 23 (2003) 179.

(Rec. 09/05/2007, Ac. 15/06/07) 